\title{
Phylogenetic relationships of Indian caecilians (Amphibia: Gymnophiona) inferred from mitochondrial rRNA gene sequences
}

\author{
Mark Wilkinson, ${ }^{\mathrm{a}, *}$ Jonathan A. Sheps, ${ }^{\mathrm{b}, 1}$ Oommen V. Oommen, ${ }^{\mathrm{c}}$ and Bernard L. Cohen ${ }^{\mathrm{b}}$ \\ a Department of Zoology, The Natural History Museum, London SW7 5BD, UK \\ ${ }^{\mathrm{b}}$ Institute of Biomedical and Life Sciences, Division of Molecular Genetics, University of Glasgow, Pontecorvo Building, 56 Dumbarton Rd, \\ Glasgow G11 6NU, Scotland, UK \\ ${ }^{\mathrm{c}}$ Department of Zoology, University of Kerala, Kariavattom 695 581, Thiruvananthapuram, Kerala, India
}

Received 27 June 2001; received in revised form 17 December 2001

\begin{abstract}
India has a diverse caecilian fauna, including representatives of three of the six currently recognized families, the Caeciliidae, Ichthyophiidae, the endemic Uraeotyphlidae, but previous molecular phylogenetic studies of caecilians have not included sequences for any Indian caecilians. Partial 12S and 16S mitochondrial gene sequences were obtained for a single representative of each of the caecilian families found in India and aligned against previously reported sequences for 13 caecilian species. The resulting alignment (16 taxa, 1200 sites, of which 288 cannot be aligned unambiguously) was analyzed using parsimony, maximum-likelihood, and distance methods. As judged by bootstrap proportions, decay indices, and leaf stabilities, well-supported relationships of the Indian caecilians are recovered from the alignment. The data (1) corroborate the hypothesis, based on morphology, that the Uraeotyphlidae and Ichthyophiidae are sister taxa, (2) recover a monophyletic Ichthyophiidae, including Indian and South East Asian representatives, and (3) place the Indian caeciliid Gegeneophis ramaswamii as the sister group of the caeciliid caecilians of the Seychelles. Rough estimates of divergence times suggest an origin of the Uraeotyphlidae and Ichthyophiidae while India was isolated from Laurasia and Africa and are most consistent with an Indian origin of these families and subsequent dispersal of ichthyophiids into South East Asia. ㅇ 2002 Elsevier Science (USA). All rights reserved.
\end{abstract}

Keywords: Evolution; Phylogeny; Biogeography; Gondwana; Vertebrates; Amphibians

\section{Introduction}

Caecilians (Gymnophiona), frogs and toads (Anura), and newts and salamanders (Caudata) are the three Orders of the extant Amphibia. Caecilians have distinctive, elongate and limbless body forms, and gymnophionan monophyly, which has never been seriously questioned, is supported by numerous morphological attributes (e.g., Nussbaum and Wilkinson, 1989). The vast majority of the approximately 160 currently recognized species are fossorial, inhabiting soils throughout much of the wet tropics. Due to their secretive habits,

\footnotetext{
${ }^{*}$ Corresponding author. Fax: +44-20-7942-5433.

E-mail address: marw@nhm.ac.uk (M. Wilkinson).

${ }^{1}$ Present address: Institute of Molecular Biology and Biochemistry, Simon Fraser University, 8888 University Drive, Burnaby, British Columbia V5A 1S6, Canada.
}

caecilians are inconspicuous members of tropical herpetofaunas and it is widely recognized that many aspects of their biology are poorly known. In contrast, Hillis (1991) concluded that the broad outlines of caecilian phylogeny were better established than phylogenies the other amphibian groups. This favorable assessment was based on analyses of morphological and life history data for the six currently recognized families of caecilians (Nussbaum and Wilkinson, 1989). However, major problems remain. For example, the largest family, the Caeciliidae, is probably paraphyletic (Nussbaum and Wilkinson, 1989; Hedges et al., 1993) and the relationships of its constituent genera to each other and to the Typhlonectidae and Scolecomorphidae have not been satisfactorily resolved with morphological data (Wilkinson, 1997).

Molecular sequence data have previously been used to infer caecilian interrelationships only by Hedges et al. 
(1993) who employed mitochondrial (mt) small (12S) and large (16S) subunit ribosomal DNA (rDNA) partial sequences from 13 species belonging to four of the six families. These were aligned with frog, salamander, and human outgroup sequences, with the distant human sequence being used to root the trees. Parsimony and distance analyses provided strong support for monophyly of the Gymnophiona, but the interrelationships of the caecilians were less compellingly resolved. Only 5 of the 11 ingroup clades were supported by bootstrap proportions greater than $70 \%$ in both parsimony and distance analyses. However, the molecular trees were mostly consistent with prevailing views of caecilian phylogeny (Nussbaum and Wilkinson, 1989) and provided useful support and, to some extent, refinement of these views. Thus, these molecular data provide a good foundation upon which to build. Hedges et al. (1993) were unable to include any representatives of two families, the African Scolecomorphidae and the Indian Uraeotyphlidae, or of any caeciliids from East Africa or India. In this report we begin to fill these gaps with sequence data from a range of Indian caecilians.

The previous lack of sequences from Indian caecilians is significant because the caecilian fauna of the subcontinent is both taxonomically and morphologically diverse and of considerable biogeographic interest. The latest taxonomic review recognized some 20 endemic Indian species, comprising over $10 \%$ of all currently recognized caecilian species (Pillai and Ravichandran, 1999). The Indian caecilian fauna includes representatives of the Ichthyophiidae (otherwise known only from Sri Lanka and South East Asia), two endemic genera of caeciliids (presumably related to the Caeciliidae of Africa, the Seychelles, and the Americas), and an endemic family, the Uraeotyphlidae. The Uraeotyphlidae was initially identified by phylogenetic studies of morphological and life history data as the sister group of a "higher" caecilian clade that included the Caeciliidae, Scolecomorphidae, and Typhlonectidae (Nussbaum, 1979, 1991; Duellman and Trueb, 1986; Hillis, 1991). In contrast, more recent morphological analyses have found strong support for the alternative hypothesis, that the Uraeotyphlidae and Ichthyophiidae are each others closest relatives (Wilkinson, 1997; Wilkinson and Nussbaum, 1996). Here, we report new $12 \mathrm{~S}$ and $16 \mathrm{~S}$ SSU rDNA partial sequences from caecilians representing each of the three families found in India. These sequences allow the first molecular tests of the alternative hypotheses for the phylogenetic relationships of the Uraeotyphlidae and of alternative biogeographic hypotheses for the distribution of the Ichthyophiidae and of ichthyophiid monophyly, and they provide an assessment of the position of Indian caeciliids within the "higher" caecilian clade.

\section{Materials and methods}

We sequenced parts of the small (12S) and large (16S) subunit mitochondrial $(\mathrm{mt})$ ribosomal RNA genes of representatives of each of the three families of caecilians that occur in India. Voucher specimens are deposited in the collection of the Department of Zoology, University of Kerala, as follows. Ichthyophiidae, Ichthyophis tricolor Annandale, MW 1712, collected near Punalur, Kollam, Kerala, by Biju Thomas, May 1995, at $08^{\circ} 58^{\prime}$ $45^{\prime \prime} \mathrm{N}, 76^{\circ} 57^{\prime} 04^{\prime \prime} \mathrm{E}, 70 \mathrm{~m}$ above sea level (asl). Uraeotyphlidae: Uraeotyphlus sp. MW 1711, collected near Vandiperiyar, Idukki, Kerala, by Thomas T. Valamparampil, May 1995 at $09^{\circ} 31^{\prime} 08^{\prime \prime} \mathrm{N}, 77^{\circ} 05^{\prime} 28^{\prime \prime} \mathrm{E}, 885 \mathrm{~m}$ asl. The specimen belongs to an undescribed species that is most similar to Uraeotyphlus malabaricus (Beddome) (D. Gower, M. Wilkinson \& O.V. Oommen, unpublished). Caeciliidae; Gegeneophis ramaswamii Taylor. Collection data as for MW 1712. No voucher specimen exists for this sample, but two specimens (MW 1713 and 1714) taken from the same locality at the same time are deposited at the University of Kerala.

Animals were killed by lethal anesthesia with MS222 (Sandoz) and samples of liver and/or muscle were preserved in 96\% ethanol. Genomic DNA was extracted and purified using standard methods (Sambrook et al., 1989). Mitochondrial rDNA sequences were amplified and sequenced by the polymerase chain reaction using specific primers (Kocher et al., 1989 [12S]; Hedges et al., 1993 [16S]). One new primer was constructed (16F2841GGG GCG ACC ACG GAG AAA ACA AAA CCT CC) to sequence the $3^{\prime}$ end of the $16 \mathrm{~S}$ rDNA fragment. Except for short regions near the $5^{\prime}$ ends, each sequence was determined from both strands with multiple redundancy and the final sequence represents a majority-rule consensus. Sequences have been deposited in GenBank (Benson et al., 1998) with accession numbers AF461136-AF461141. The sequence alignment (with mask) is available as a NEXUS file (Maddison et al., 1997) from http://www.nhm.ac.uk/zoology/home/wilkinson.htm.

The newly determined sequences were added to an alignment of concatenated partial $12 \mathrm{~S}$ and $16 \mathrm{~S}$ caecilian sequences kindly provided in digital form together with their alignment mask, by S. Blair Hedges (Hedges et al., 1993). Data from three distantly related outgroups were removed and the alignment mask was adjusted to eliminate sites regarded as potentially misaligned solely because of these outgroups. The sequence of the rhinatrematid caecilian Epicrionops marmoratus Taylor was designated as a single outgroup and used to root our trees. This rooting is justified because the hypothesis that the Rhinatrematidae is the sister group of all other caecilians is supported by previous molecular analyses (Hedges et al., 1993) and by diverse morphological synapomorphies (Nussbaum, 1977, 1979; Wilkinson, 
1992, 1996) and is the best-supported caecilian phylogenetic relationship yet proposed (Wilkinson, 1997). Hedges et al. (1993) reported their rhinatrematid sequence as Epicrionops sp. Examination of the voucher suggests that it can be tentatively assigned to E. marmoratus.

Parsimony, maximum-likelihood (ML), and distance analyses were performed with PAUP*4b4 (Swofford, 1998). LogDet and maximum-likelihood distance (MLD) analyses used the minimum-evolution objective function. ML and MLD analyses used the general timereversible model, with empirical base frequencies. Rate matrix parameters, gamma distribution shape parameters, and the proportions of invariant sites were estimated iteratively through full likelihood evaluation of optimal trees, beginning with the most parsimonious tree, until they stabilized. Iterative optimization was also used to estimate the proportion of invariant sites in the LogDet analysis. Alignment gaps were treated as missing data. Tree searches were either branch-and-bound (parsimony) or heuristic with 10 random addition sequences and TBR branch swapping. A parsimony PTP test (Faith and Cranston, 1991) was used to evaluate the data (100 random permutations). Support for clades was measured with bootstrap proportions (Felsenstein, 1985) (100 pseudoreplicates) and decay indices determined by enforcing converse topological constraints (Bremer, 1998). The significance of parsimony length differences between trees were assessed using nonparametric Templeton tests (Templeton, 1983).

For saturation analyses, pairwise transition and transversion differences were estimated (after excluding potentially misaligned sites) in PAUP* and scattergrams plotted of both transitions versus transversions and each versus pairwise maximum-likelihood distances. Linear, power, logarithmic, exponential, and second-order polynomial curves were fitted to the data and selected for the highest value of $r^{2}$ (to two significant figures). Relative rate tests based on the relationships recovered in the most parsimonious tree were performed with RRTree (Robinson et al., 1998).

Leaf stabilities were determined using RadCon (Thorley and Page, 2000) from sets of bootstrap trees generated in corresponding parsimony, MLD, and LogDet analyses. Leaf stabilities were based on the bootstrap difference measure introduced by Thorley and Wilkinson (1999) but calculated for quartets rather than triplets, corresponding to an unrooted rather than a rooted analysis and thereby allowing assessment of the stability of the rooting on E. marmoratus.

Speculative estimates of dates of divergence were based on the phylogenetic results. For a given node we call any pair of taxa whose most recent common ancestor is that node a corresponding nodal pair. A single node was considered to be at least as old as the separation of the Madagascar-India-Seychelles sepa- ration from Afro-American Gondwanaland, dated at 130 million years ago (MYA) (Smith et al., 1994) and used for calibration (see below). A point estimate of the rate of divergence was determined from the average of the ML distances of the corresponding nodal pairs, divided by the assumed divergence time. Point estimates of the dates of divergence of other nodes were given by the average of the pairwise distance of all corresponding nodal pairs divided by the estimated rate of divergence. This is equivalent to the average distance method of Kumar and Hedges (1998). More conservative interval estimates were calculated analogously using slowest and fastest rate estimates given by the smallest and the greatest distances of the nodal pairs about the calibration point, respectively. For a given node, minimum and maximum divergence time estimates were given by the smallest and the greatest distances of the corresponding nodal pairs divided by the fastest and slowest rate estimates, respectively. Siphonops annulatus was excluded from these estimations because of its performance in relative rates tests (see below).

\section{Results}

All PCR amplifications from genomic DNA yielded single products of the expected size which, on sequencing, contained negligible levels of site ambiguity. There is no reason, either in our work or in that previously reported (Hedges et al., 1993), to suspect that any of the data could have been derived from nuclear copies of mitochondrial sequences.

The aligned sequences spanned 1200 sites, of which 288 were excluded as potentially misaligned. Of the remaining 912 sites, 483 were invariant and of these, 154 sites were parsimony uninformative, leaving 275 parsimony-informative sites. There was no significant variation in base composition across the alignment after excluding potentially misaligned sites $\left(\chi^{2}\right.$ tests for homogeneity, $P=0.98$, df $=45$ ). In 16 pairwise $\chi^{2}$ tests between the outgroup Epicrionops and one other taxon, significant heterogenenity was found in only one case (Dermophis, $P=0.016$ ). The three Indian taxa did not differ significantly in base composition from one another nor from Dermophis $(P>0.34)$. In saturation analyses the scattergram of transitions versus transversions was best fitted by a power curve that departed only slightly from linearity $\left(r^{2}=0.761\right)$. Similarly, when plotted as a function of maximum-likelihood distances, transversions showed only a very slight departure from linearity $\left(r^{2}=0.943\right)$. Transitions appeared to be only slightly more saturated $\left(r^{2}=0.917\right)$. Thus, neither base composition variation nor saturation due to multiple substitutions are expected to be major obstacles to phylogenetic inference. 
Relative rate tests revealed that $S$. annulatus evolved more slowly than all other taxa $(P=0.008)$, but there were no significant differences in rate $(P>0.05)$ between any other taxa or within any other clades. Thus, neither long- nor short-branch attraction are likely to represent serious problems.

The data have a parsimony PTP of 0.01 , allowing rejection of the null hypothesis that the data contain no more congruence than expected by chance alone. Parsimony analysis of the full data yielded a single tree (Fig. 1A). The interrelationships of the taxa represented in Hedges et al.'s original alignment are mostly the same as in Hedges et al.'s trees (see below). The relationships of the Indian caecilians are well resolved. G. ramaswamii is recovered as the closest relative of the Seychellean caeciliid clade (Hypogeophis + Grandisonia + Praslinia), I. tricolor is recovered as the closest relative of its South East Asian congener I. bannanicus, and Uraeotyphlus is recovered as the closest relative of these Ichthyophis species. The same relationships are found in the ML and distance analyses (Fig. 1B) with differences only in the interrelationships within the Seychellean clade (largely concerning the interelationships of Hypogeophis rostratus and the species of Grandisonia) and in the position of the south American caeciliid $S$. annulatus.

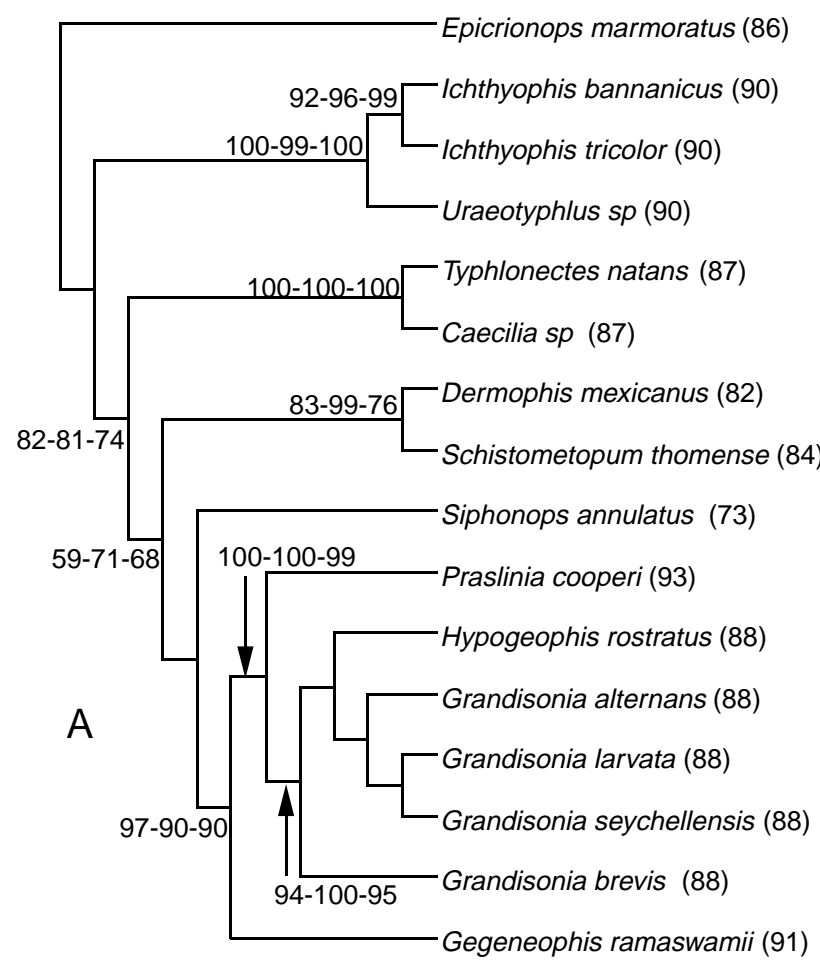

The relationships of the Indian caecilians are also well supported as judged by bootstrap proportions. The lowest bootstrap proportions $(90 \%)$ are for the grouping of $G$. ramaswamii with the Seychelles caecilians in the distance analyses. With parsimony analysis this relationship is better supported $(\mathrm{BP}=97 \%)$. The corresponding decay indices are +8 for the pairing of $I$. tricolor and I. bannanicus, +10 for the grouping of Gegeneophis with the Seychellean caeciliids, and +16 for the clade comprising Uraeotyphlus and Ichthyophis. The latter relationship appears particularly robust with maximal or near-maximal bootstrap proportions in all analyses and decay indices that are exceeded only by the pairing of Typhlonectes natans and Caecilia sp. (+21). Judged by the Templeton test the most parsimonious tree has a significantly better fit to the data $(P \leqslant 0.1)$ than alternatives that do not include the UraeotyphlusIchthyophis clade or that do not include the $G$. ramaswamii + Seychelles caeciliid clade. Significance was otherwise found only in tests of the pairing of T. natans and Caecilia sp. and of the monophyly of the Seychelles caeciliids. For the Ichthyophis clade the test result is not significant $(P=0.1441)$.

Leaf stabilities, calculated from the parsimony, MLD, and LogDet bootstrap analyses all identified

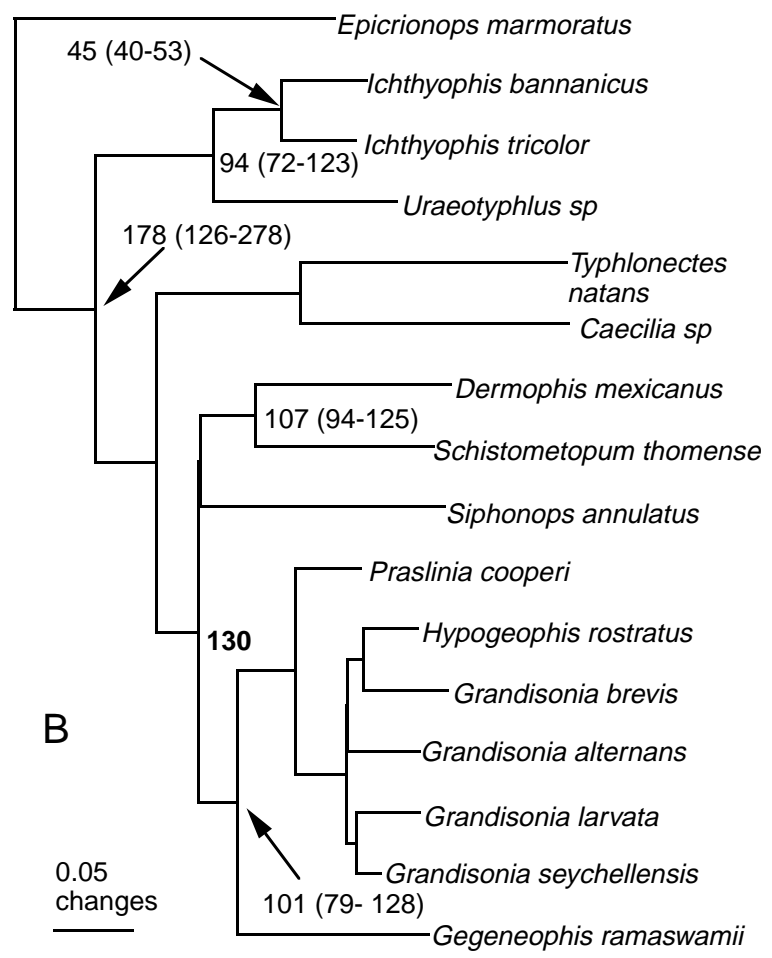

Fig. 1. (A) Single most parsimonious tree (length $=1100 / 913$ steps, consistency index $=0.566 / 0.478$, retention index $=0.490 / 0.490$ with all characters/ parsimony-informative characters only). Bootstrap proportions of clades determined using parsimony, MLD, and LogDet are indicated adjacent to the corresponding internal branches where these values exceed $50 \%$. Numbers in parentheses are averages of the leaf stabilities determined from parsimony, MLD, and LogDet bootstrap analyses and scaled between zero and 100. (B) Single maximum-likelihood phylogram $(-\operatorname{lnL}=5933.3111$, proportion of invariant sites $=0.1152$, gamma shape parameter $=0.4242$ ). Numbers are point and, in parentheses, interval estimates of divergence dates of selected nodes, except the number in boldface which is the assumed 130 MYA date of divergence of the Indian-Seychellean caeciliids from the African and Central American species Schistometopum thomense and Dermophis mexicanus. 
Praslinia cooperi and $S$. annulatus as the most and least stable, respectively (Fig. 1). Leaf stabilities for the Indian taxa are higher than the average (87), indicating that their positions are among the relatively best supported. Leaf stability of E. marmoratus is close to the average, indicating no especial instability in the root.

\section{Discussion}

\subsection{Relationships of Indian caecilians}

Nussbaum (1979) used morphology to investigate the relationships among 13 caecilian genera including representatives of all the then recognized families. His analysis recovered Uraeotyphlus as the sister genus of a higher caecilian clade comprising the caeciliids (including Gegeneophis), the typhlonectids, and the scolecomorphids. This pattern of relationships was also found in the subsequent derivative analyses of Duellman and Trueb (1986) and Hillis (1991) that used composite family-level terminal taxa. However, Hillis (1991) also found that the position of Uraeotyphlus was the least well supported. Wilkinson and Nussbaum (1996) conducted an expanded analysis including additional taxa and characters and found strong support for the alternative hypothesis that Uraeotyphlus is more closely related to ichthyophiids than to other caecilians. This was also among the strongest supported relationships from analysis of the most extensive nonmolecular data to date (Wilkinson, 1997). Our analyses show that molecular data also provide strong support for this hypothesis, and, in our view, the congruence of morphological and molecular data is compelling.

Notwithstanding the nonsignificance using the Templeton test, the pairing of I. tricolor with its congener $I$. bannanicus is also well supported by the molecular data and is entirely consistent with current views of caecilian phylogeny. However, given that our analysis includes only these two ichthyophiids, a single uraeotyphlid, and no members of the other ichthyophiid genus, Caudacaecilia, it represents only a minimal molecular test of the monophyly of Ichthyophis or of the Ichthyophiidae.

The relationships of the Indian caecililiid Gegeneophis are well supported by the molecular data and are, as expected, with the non-Indian caeciliids. In particular, the molecular data strongly supports a relationship between Gegeneophis and the clade of Seychellean caecilians. This is in contrast to the most recent morphological study in which Gegeneophis was recovered as a member of a group of caeciliids characterized by reduced visual systems and with no particular association with the Seychellean caeciliids (Wilkinson, 1997). In Gegeneophis, the eye and associated structures are greatly reduced and the orbit is covered with bone (Ramaswami, 1943; Wake, 1985). Such features occur in a number of other caeciliid genera and have generally been viewed as associated with enhanced burrowing performance. Wilkinson (1997) suggested that the apparent support for the close relationships of Gegeneophis and other caeciliid genera with vestigial eyes might be due to concerted homoplasy in the rudimentation of the visual system, a conclusion supported by O'Keefe and Wagner (2001). Molecular data could provide a useful independent assessment of these views. Unfortunately, no other caecilians with rudimentary visual systems could be included in this study. Although the precise membership of the clade of caeciliids with reduced visual systems in morphological analyses differed depending on the method of analysis, Gegeneophis was consistently recovered as most closely related to the East African caeciliid Boulengerula (Wilkinson, 1997), making the latter genus the obvious target for future molecular studies.

\subsection{Biogeography and times of divergence}

It seems reasonable to assume that, like other amphibians, caecilians are incapable of long-distance transoceanic dispersal (Nussbaum, 1984). Hence their global patterns of distribution are expected to reflect terrestrial dispersal and continental drift. Given the primarily Gondwanan distribution of extant caecilians, the origin of the South East Asian caecilian fauna is, as noted by Hedges et al. (1993), one of the major questions in caecilian biogeography. The Ichthyophiidae is the only caecilian family with representatives in South East Asia and is otherwise known from South Asia (India and Sri Lanka). Duellman and Trueb (1986) hypothesized that the presence of caecilians in South East Asia is attributable to the dispersal of ichthyophiids from the Indian Plate subsequent to its collision with Asia. We refer to this as the Out of India hypothesis. Hedges et al. (1993, p. 74) proposed an alternative biogeographic hypothesis in which "ichthyophiids (and possibly uraeotyphlids) became isolated in Laurasia either (1) when the Tethys Sea separated Pangaea into Laurasia and Gondwanaland in the Jurassic, or (2) by dispersal from nearby Gonwanaland in the late Jurassic or early Cretaceous." They noted that this hypothesis implies that ichthyophiids dispersed into India from South East Asia and we refer to it as the Out of Laurasia hypothesis. More recently, Feller and Hedges (1998) proposed that the origin of salamanders and caecilians was associated with Gondwanan-Laurasian vicariance based on analysis of sequences of four mitochondrial genes of nine amphibians, including three caecilians, and they suggested a Gondwanan origin for ichthyophiids.

Hedges et al. (1993, p. 73) interpreted the Out of India hypothesis as implying either that ichthyophiids and uraeotyphlids were restricted to the Indian Plate or 
that they had a broader Gondwanan distribution and subsequently became extinct from all southern areas, and they considered that "Both of these alternatives seem unlikely." However, like the Out of India hypothesis, the Out of Laurasia hypothesis also requires either a restricted distribution of the ichthyophiids (within Pangaea or Gondwana) or their disappearance from large areas. In our view, that many groups have restricted distributions and that the frequency of regional extinctions is unknown imply thatbiogeographic hypotheses are not implausible by virtue of their entailing such events. In the present case, these implications provide no basis for choosing between the Out of India and the Out of Laurasia hypotheses.

Molecular data might help discriminate between the alternative biogeographic hypotheses by providing evidence of relationships or by allowing estimation of divergence dates. Our estimates of divergence times are based on the assumption that the separation of the caeciliids of the Seychelles and India from their closest relatives in Africa and the New World occurred at or prior to the separation of Madagascar-India-Seychelles from Afro-American Gondwana at about 130 MYA (Smith et al., 1994). The fossil record of caecilians is insufficient to provide any basis for calibration. We view our estimates as highly speculative given that they are likely subject to many of the problems discussed by Hillis et al. (1996). In addition, the calibration uses the minimum age of a node that could be much older so that the rate may have been overestimated and divergence dates correspondingly underestimated. Similarly, our sampling of African and South American taxa is sparse and we cannot exclude possible relationships that would invalidate our calibration. For example, if G. ramaswamii is actually more closely related to any of the other caeciliids with reduced visual systems than to the Seychelles caeciliids then this would invalidate our speculative estimates of divergence times. Finally, the node used for the calibration is not as well supported as we would like. However, we agree with Nei and Kumar (2000) that so little is known of dates of divergence that even rough estimates may be useful and we consider our interval estimates to be fairly conservative.

Hedges et al. (1993) based their Out of Laurasia hypothesis on morphological and molecular phylogenetics, including the immunological study of Hass et al. (1993), which they interpreted as indicating that "the Ichthyophiidae and Uraeotyphlidae are old groups whose origin must have predated the breakup of Gondwanaland." Our date estimates are consistent with such an ancient origin for the ichthyophiid-uraeotyphlid clade as a whole. In contrast, the Uraeotyphlidae and Ichthyophiidae do not appear to be such ancient groups. We estimate the divergence of ichthyophiids and uraeotyphlids to have been between 72 and 123 MYA, which is after the isolation of the Madagascar-India-Seychelles land mass in the initial breakup of Gondwana and prior to any contact between India and Laurasia. Given this divergence date, and barring any transoceanic dispersal, the Out of India hypothesis implies that the Ichthyophiidae and Uraeotyphlidae originated on an isolated landmass including India and the Seychelles either before or after their separation from Madagascar. Thus no dispersal is required to account forthe presence of uraeotyphlids in peninsular India. In contrast, the Out of Laurasia hypothesis implies an origin of Uraeotyphlidae and Ichthyophiidae elsewhere (in Laurasia or an associated Gondwanan fragment) with subsequent dispersal into India and, in the case of the Uraeotyphlidae, extinction elsewhere. Bossuyt and Milinkovitch (2001) recently provided evidence for multiple radiations of subfamilies of ranine frogs "Out of India" and, although we must remain cautious about our estimates of divergence times, the caecilian molecular data appear to fit this pattern. Note, however, that given the early origin of the ichthyophiid-uraeotyphlid clade, the absence of members of this lineage from Afro-American Gondwanan landmasses remains unexplained.

The distribution of the large and heterogenous family Caeciliidae is almost entirely Gondwanan, suggesting a Gondwanan ancestry for the Indian and Seychellean caeciliids. That the Seychellean and Indian caeciliids are more closely related to each other than to any of the South American and African caeciliids is concordant with the sequence of the breakup of Gondwana and consistent with their common ancestral lineage having been isolated on India-Madagascar-Seychelles. This underpins our calibration. Considering that the earliest putative stem group caecilian dates from the Jurassic of North America (Jenkins and Walsh, 1993) and that caecilians "are a very ancient group," Savage and Wake (2001, p. 58) state that the primarily Gondwanan distribution of caecilians "implies an early Pangaean distribution" with the central American DermophisGymnopis clade "the only remaining definitive Laurasian representatives." In our analyses, as in that of Hedges et al. (1993), Dermophis is placed within several otherwise strictly Gondwanan clades, with a strongly supported close relationship to the West African Schistometopum thomense. If both Savage and Wake (2001) and our tree (Fig. 1) are correct then much of the diversification of caeciliid caecilians must have occurred before the breakup of Pangea and our estimated divergence dates would be invalid. Instead, we suggest that Schistometopum and the Dermophis-Gymnopis lineages were separated by African-South American vicariance and that the latter dispersed into Central America from South America. Our speculative estimated divergence of Dermophis and Schistometopum of between 94 and 125 MYA is broadly compatible with this hypothesis. 


\section{Acknowledgments}

We are grateful to Biju Thomas and Thomas Valamparampil for collecting the caecilian specimens used in this study and to Blair Hedges for providing an electronic copy of his alignment. We thank Peter Foster for helpful advice on the phylogenetic analysis and David Gower for comments on the manuscript. This work was supported in part by NERC GST/02/832.

\section{References}

Benson, D.A.etal, 1998. GenBank. Nucleic Acids Res. 26, 1-7.

Bremer, K., 1998. The limits of amino acid sequence data in angiosperm phylogenetic reconstruction. Evolution 42, 795-803.

Bossuyt, F., Milinkovitch, M.C., 2001. Amphibians as indicators of Early Tertiary Out of India dispersal of vertebrates. Science 292, 93-95.

Duellman, W.E., Trueb, L., 1986. Biology of Amphibians. McGrawHill, New York.

Faith, D.P., Cranston, P.S., 1991. Could a cladogram this short have arisen by chance alone?: On permutation tests for cladistic structure. Cladistics 7, 1-28.

Feller, A.E., Hedges, S.B., 1998. Molecular evidence for the early history of living amphibians. Mol. Phylogenet. Evol. 9, 509-516.

Felsenstein, J., 1985. Confidence limits on phylogenies: An approach using the bootstrap. Evolution 39, 783-791.

Hass, C.A., Nussbaum, R.A., Maxson, L.R., 1993. Immunological insights into the evolutionary history of caecilians (Amphibia: Gymnophiona): Relationships of the Seychellean caecilians and a preliminary report on Family-level relationships. Herpetol. Monogr. 7, 56-63.

Hedges, S.B., Nussbaum, R.A., Maxson, L.R., 1993. Caecilian phylogeny and biogeography inferred from mitochondrial DNA sequences of the 12S rRNA and 16S rRNA genes (Amphibia: Gymnophiona). Herpetol. Monogr. 7, 64-76.

Hillis, D.M., 1991. The phylogeny of amphibians: Current knowledge and the role of cytogenetics. In: Sessions, S.K., Green, D.M. (Eds.), Amphibian Cytogenetics and Evolution. Academic Press, San Diego, pp. 7-31.

Hillis, D.M., Mable, B.K., Moritz, C., 1996. Applications of molecular systematics. In: Hillis, D.M., Moritz, C., Mable, B.K. (Eds.), Molecular Systematics. Sinauer, Sunderland, MA, pp. 515-543.

Jenkins, F.A., Walsh, D.M., 1993. An Early Jurassic caecilian with limbs. Nature 365, 246-249.

Kocher, T.D. et al., 1989. Dynamics of mitochondrial DNA evolution in animals: Amplification and sequencing with conserved primers. Proc. Natl. Acad. Sci. USA 89, 6196-6200.

Kumar, S., Hedges, S.B., 1998. A molecular timescale for vertebrate evolution. Nature 392, 917-920.

Maddison, D.R., Swofford, D.L., Maddison, W.P., 1997. NEXUS: An extensible file format for systematic information. Syst. Biol. 46, 590-621.

Nei, N., Kumar, S., 2000. Molecular Evolution and Phylogenetics. Oxford University Press, Oxford.
Nussbaum, R.A., 1977. Rhinatrematidae: A new family of caecilians (Amphibia: Gymnophiona). Occas. Pap. Mus. Zool. Univ. Mich. $682,1-30$.

Nussbaum, R.A., 1979. The taxonomic status of the caecilian genus Uraeotyphlus Peters. Occas. Pap. Mus. Zool. Univ. Mich. 687, 120.

Nussbaum, R.A., 1984. Amphibians of the Seychelles. In: Stoddart, D.R. (Ed.), Biogeography and Ecology of the Seychelles Islands. Junk, The Hague, pp. 379-415.

Nussbaum, R.A., 1991. Cytotaxonomy of caecilians. In: Sessions, S.K., Green, D.M. (Eds.), Amphibian Cytogenetics and Evolution. Academic Press, San Diego, pp. 22-76.

Nussbaum, R.A., Wilkinson, M., 1989. On the classification and phylogeny of caecilians (Amphibia: Gymnophiona), a critical review. Herpetol. Monogr. 3, 1-42.

O'Keefe, F.R., Wagner, P.J., 2001. Inferring and testing hypotheses of cladistic character dependence using character compatibility. Syst. Biol. 50, 657-675.

Pillai, R.S., Ravichandran, M.S., 1999. Gymnophiona (Amphibia) of India: A taxonomic study. Rec. Zool. Surv. India Occas. Pap. 172, $1-117$.

Ramaswami, L.S., 1943. An account of the head morphology of Gegenophis [sic] carnosus Beddome, Apoda. J. Mysore Univ. 3, 205-220.

Robinson, M. et al., 1998. Sensitivity of the relative-rate test to taxonomic sampling. Mol. Biol. Evol. 15, 1091-1098.

Sambrook, J., Fritsch, E.F., Maniatis, T., 1989. Molecular Cloning: A Laboratory Manual. Cold Spring Harbor Laboratory press, Cold Spring Harbor, NY.

Savage, J.M., Wake, M.H., 2001. Reevaluation of the status of the taxa of Central American caecilians (Amphibia: Gymnophiona) with comments on their origin and evolution. Copeia 2001, 52-64.

Smith, A.G., Smith, D.G., Funnell, B.M., 1994. Atlas of Mesozoic and Cenozoic Coastlines. Cambridge University Press, Cambridge.

Swofford, D.L., 1998. PAUP*: phylogenetic analysis using parasimony. Test version $4 \mathrm{~b} 4$. Sinauer, Sunderland, MA.

Templeton, A.R., 1983. Phylogenetic inference from restriction endonuclease cleavage site maps with particular reference to the evolution of humans and the apes. Evolution 37, 221-244.

Thorley, J.L., Page, R.D.M., 2000. RadCon: Phylogenetic tree comparison and consensus. Bioinformatics 16, 486-487.

Thorley, J.L., Wilkinson, M., 1999. Testing the phylogenetic stability of early tetrapods. J. Theor. Biol. 200, 343-344.

Wake, M.H., 1985. The comparative morphology and evolution of the eyes of caecilians (Amphibia: Gymnophiona). Zoomorphology 105, 277-295.

Wilkinson, M., 1992. The phylogenetic position of the Rhinatrematidae (Amphibia: Gymnophiona): Evidence from the larval lateral line system. Amphibia-Reptilia 13, 74-79.

Wilkinson, M., 1996. The heart and aortic arches of rhinatrematid caecilians (Amphibia: Gymnophiona). Zool. J. Linn. Soc. 118, 135150 .

Wilkinson, M., 1997. Characters, congruence and quality: A study of neuroanatomical and traditional data in caecilian phylogeny. Biol. Rev. 72, 423-470.

Wilkinson, M., Nussbaum, R.A., 1996. On the phylogenetic position of the Uraeotyphlidae (Amphibia: Gymnophiona). Copeia 1996, $550-562$. 